\title{
Demographic, clinical, electrocardiographic and echocardiographic characteristics of patients hospitalized with COVID-19 and cardiac disease at a tertiary hospital, South Africa
}

\author{
Ruchika Meel $^{1} \wedge$, Sarah A. Van Blydenstein ${ }^{2}$ \\ ${ }^{1}$ Division of Cardiology, Department of Internal Medicine, Chris Hani Baragwanath Academic Hospital, Faculty of Health Sciences, University of \\ the Witwatersrand, Johannesburg, South Africa; ${ }^{2}$ Division of Pulmonology, Department of Internal Medicine, Chris Hani Baragwanath Academic \\ Hospital, Faculty of Health Sciences, University of the Witwatersrand, Johannesburg, South Africa \\ Contributions: (I) Conception and design: R Meel; (II) Administrative support: Both authors; (III) Provision of study materials or patients: Both \\ authors; (IV) Collection and assembly of data: Both authors; (V) Data analysis and interpretation: Both authors; (VI) Manuscript writing: Both \\ authors; (VII) Final approval of manuscript: Both authors. \\ Correspondence to: Dr. Ruchika Meel, MBChB, MMED, Cert Cardiology (SA), PhD, FEACVI. 17 North Road, Hyde Park Village, House No. 17, \\ Johannesburg, 2196, South Africa. Email: ruchikameel@gmail.com.
}

Background: Coronavirus associated disease 2019 (COVID-19) is associated with higher morbidity and mortality in patients with cardiovascular disease. There is a paucity of data regarding COVID-19 and cardiac disease from Africa. We aimed to describe the demographic, clinical, electrocardiographic and echocardiographic characteristics of patients with COVID-19 and cardiac disease at a tertiary hospital in South Africa.

Methods: This was a retrospective cross-sectional descriptive study (Aug 2020 to March 2021) of 200 patients with COVID-19 and confirmed cardiac disease, conducted at Chris Hani Baragwanath. Demographic, clinical, electrocardiographic and echocardiographic characteristics were systematically collected.

Results: Majority (86\%) of patients were Africans with mean age $56.4 \pm 15.6$ years $(57.5 \%$ females). Fifty three percent were unemployed and 28\% were pensioners. Main comorbidities were hypertension (69.5\%), diabetes mellitus (31.5\%) and human immunodeficiency virus (HIV) (22.5\%). Majority of the patients were overweight or obese (65.5\%). All except 8 patients were on chronic medication. Dyspnoea on admission was noted in $88.5 \%$ of patients. Seventy nine percent of patients had abnormal chest X-Ray. Frequently documented electrocardiography findings were sinus tachycardia (63\%) and atrial fibrillation, noted in $7 \%$ of patients. The most common indication for echocardiography was heart failure (30\%). Severe left ventricular dysfunction was noted in $21.5 \%$. Features of pulmonary hypertension were present in $45.5 \%$. The right ventricle was enlarged in $59 \%$ of patients, and functional tricuspid regurgitation was noted in $54.5 \%$. The most common diagnoses were hypertensive heart disease with preserved ejection fraction $(35.8 \%)$, cardiomyopathies $(20 \%)$, cor pulmonale (15.7\%), acute coronary syndrome $(6.5 \%)$, infective endocarditis $(5.5 \%)$ and valvular heart disease $(2.5 \%)$. Echocardiography modified management in $53 \%$ of cases. An inhospital mortality of $17.5 \%$ was noted. On multivariate logistic regression analysis sinus tachycardia was the most important independent predictor of mortality (odds ratio, OR: 2.52, 95\% confidence interval, CI: 1.08-5.85, $\mathrm{P}=0.03)$.

Conclusions: Most patients were obese females with underlying hypertension. Echocardiography altered management in about half the patients. Mortality amongst this cohort of patients was high and were predominantly males.

$\wedge$ ORCID: 0000-0002-1405-4259. 
Keywords: Coronavirus associated disease 2019 (COVID-19); echocardiography, cardiac disease; Africa

Submitted Jul 25, 2021. Accepted for publication Sep 29, 2021.

doi: $10.21037 / \mathrm{cdt}-21-459$

View this article at: https://dx.doi.org/10.21037/cdt-21-459

\section{Introduction}

Coronavirus associated disease 2019 (COVID-19) has been shown to be an important cause of morbidity and mortality especially in patients with established cardiovascular disease $(1,2)$. Studies from China have shown that there is a high prevalence of cardiovascular disease, hypertension and diabetes amongst symptomatic COVID-19 patients (3). Most of patients present with lung involvement (4). However, myocardial involvement may occur in isolation $(5,6)$. COVID-19 has been shown to have a myriad of cardiovascular manifestations such as myocardial infarction, myocarditis, takotsubo cardiomyopathy and cardiac tamponade. COVID-19 can affect the heart in a direct or indirect manner. Histopathology studies have shown presence of virions in the myocardial tissue (7). Systemic inflammatory response to the virus results in a cytokinemediated storm culminating in multi-organ failure, including the heart. Echocardiography has proven to be a useful diagnostic tool in COVID-19 pandemic (8). Its easy access and portability, and permits bedside evaluation of the heart even in the most resource limited setting. Furthermore, hand-held echocardiography has been found to be a good alternative to standard echocardiography in patients with SARS-CoV-2 infection (9). Recent global echocardiography survey reported cardiac abnormalities in half of patients with COVID-19 (10). However, in the aforementioned study data from the African sub-continent was limited. Herein, we aim to describe demographic, clinical, electrocardiographic and echocardiographic characteristics of patients with cardiac disease and COVID-19 from a single tertiary referral center in South Africa.

We present the following article in accordance with the STROBE reporting checklist (available at https://dx.doi. org/10.21037/cdt-21-459).

\section{Methods}

This was a retrospective, single center, descriptive study of 200 patients with cardiac disease and COVID-19, performed at Chris Hani Baragwanath hospital between the period
August 2020 to March 2021. All data was collected from patient's files. Clinical, demographic and echocardiographic characteristics of patients that required echocardiographic imaging as part of their clinical care were documented. During the study period 215 consecutive inpatients with clinical indication for echocardiography and laboratory confirmed COVID-19 infection were scanned according to a prespecified protocol (6). All patients over the age of 18 with COVID-19 infection confirmed by molecular testing, using reverse transcriptase polymerase chain reaction (RTPCR) analysis, cardiac disease and adequate imaging quality were included in the study. Twelve patients had normal echocardiograms and were excluded for the purpose of the current study. Three were excluded due to very poor imaging quality. Qualitative echocardiographic data was recorded by an experienced imaging cardiologist using a handheld GE VSCAN ultrasound machine. A detailed echocardiogram was performed only when deemed necessary.

\section{Statistical analysis}

Statistical analysis was performed with Statistica ${ }^{\circledR}$, version 13.5, series 0414 for Windows ${ }^{\circledR}$. Continuous variables were expressed as mean $\pm \mathrm{SD}$ or median (interquartile range). Student's $t$-test or Mann-Whitney $U$ test were used to compare continuous variables. Categorical variables were evaluated by chi-square and Fisher's exact test when necessary. Univariate and multivariate logistic regression analyses were used to identify possible independent determinants of mortality. The independent variables with a $P$ value of $\leq 0.1$ on univariate analysis and variables that had clinical significance were tested in the multivariate model. Any missing echocardiographic data or laboratory data were excluded from analysis.

\section{Ethical statement}

The study was conducted in accordance with the Declaration of Helsinki (as revised in 2013). The study was approved by the ethics committee of University of the Witwatersrand (M200678). Because of the retrospective 
Table 1 Baseline characteristics of study patients

\begin{tabular}{|c|c|}
\hline Variable & Values (total $n=200$ ) \\
\hline Age (years) & $56.4 \pm 15.6$ \\
\hline Females & $115(57.5)$ \\
\hline Systolic blood pressure $(\mathrm{mmHg})$ & $129[112-150]$ \\
\hline Diastolic blood pressure $(\mathrm{mmHg})$ & $77.5(65-90.5)$ \\
\hline Heart rate (beats/min) & $100.07(23.45)$ \\
\hline Respiratory rate (breaths/min) & 22 [20-25] \\
\hline Admission room air saturation of oxygen (\%) & 87 [77-92] \\
\hline \multicolumn{2}{|l|}{ Ethnicity } \\
\hline African & $173(86.5)$ \\
\hline Indian & $7(3.5)$ \\
\hline Caucasian & $5(2.5)$ \\
\hline Mixed race & $15(7.5)$ \\
\hline \multicolumn{2}{|l|}{ Body habitus } \\
\hline Overweight/obese & $131(65.5)$ \\
\hline Morbidly obese & $7(3.5)$ \\
\hline \multicolumn{2}{|l|}{ Employment status } \\
\hline Employed & $37(18.5)$ \\
\hline Unemployed & $107(53.5)$ \\
\hline Pensioner & $56(28)$ \\
\hline \multicolumn{2}{|l|}{ Co-morbidities } \\
\hline Pre-existing cardiac disease & $26[13]$ \\
\hline Pre-existing lung disease & 36 [18] \\
\hline Human immunodeficiency virus & $45(22.5)$ \\
\hline Hypertension & 139 (69.5) \\
\hline Diabetes mellitus & $63(31.5)$ \\
\hline \multicolumn{2}{|l|}{ Medication } \\
\hline ACEI/ARB & $87(43.5)$ \\
\hline Beta blocker & $54[27]$ \\
\hline Diuretics & $127(63.5)$ \\
\hline Statins & $21(10.5)$ \\
\hline Aldosterone receptor antagonist & $24[12]$ \\
\hline Calcium channel blockers & 66 [33] \\
\hline Anti-diabetic medication & $50[25]$ \\
\hline Anti-retroviral & 38 [19] \\
\hline
\end{tabular}

Data are presented as mean \pm SD or median (IQR) or $n(\%)$. $\mathrm{ACEl} / \mathrm{ARB}$, angiotensin converting enzyme inhibitor/angiotensin receptor blocker. nature of the study, the requirement for informed consent was waived.

\section{Results}

\section{The baseline characteristics of the study patients}

The mean age was $56.4 \pm 15.6$ years and most were African females. Most of the patients were obese (65.5\%) and had hypertension $(69.5 \%)$. Other co-morbidities included pulmonary tuberculosis (9\%), chronic kidney disease $(10 \%)$, malignancy $(3 \%)$ and chronic obstructive pulmonary disease (5\%) and autoimmune disease (1\%). Eight patients did not have known co-morbidities. Half of the patients were unemployed. Most patients had concurrent lung involvement (79\%) with moderate or severe covid pneumonia. The most common symptom on presentation was shortness of breath (88.5\%) followed by cough (62\%), chest pain $(34.5 \%)$, fatigue $(29.5 \%)$ and $17 \%$ were febrile (Table 1).

\section{Blood biochemistry tests of study patients}

Markers of inflammation and coagulation such as C-reactive protein, ferritin and $\mathrm{D}$-dimer were elevated in all patients. Evidence of myocardial injury was noted in 122 patients. Thirty patients had a highly sensitive troponin $\mathrm{T}$ value of less than $14 \mathrm{ng} / \mathrm{L}$. Thirteen patients were diagnosed with acute coronary syndrome (Table 2).

\section{The main electrocardiographic findings}

The main electrocardiographic findings are depicted in Table 3. Sinus tachycardia was noted in the majority (63\%). Sinus bradycardia was noted in $10(5 \%)$ patients who were all above age of 50 years. Atrial fibrillation was the most common tachyarrhythmia (7\%). Ten patients with atrial fibrillation/flutter had underlying hypertensive heart disease. All patients were above the age of 40 years. There was a negative correlation between age and heart rate (Figure 1). Only one patient was less than age of 32 years and presented with first episode of SVT and no underlying structural heart disease. ST segment depression was a frequent finding present in $23 \%$ of patients. $\mathrm{T}$ wave inversion in the anterolateral leads was noted in $14,5 \%$ of cases. Prolonged QT interval was noted in only 2 patients.

The main indications for echocardiograms comprised heart failure $(30 \%)$, suspected hypertensive heart 
Table 2 Blood biochemistry of study patients

\begin{tabular}{|c|c|c|}
\hline Variable & Value & Normal range \\
\hline Lymphocytes & $1.5(0.7-2.7)$ & $0.63 \times 10^{9} / \mathrm{L}$ \\
\hline Neutrophils & $7.2(4.6-12.5)$ & $1.98 \times 10^{9} / \mathrm{L}$ \\
\hline Haemoglobin & $12.9(11.2-14.2)$ & $12.1-16.3 \mathrm{~g} / \mathrm{dL}$ \\
\hline Urea & $6.4(4.6-11.7)$ & $2.6-7.0 \mathrm{mmol} / \mathrm{L}$ \\
\hline Creatinine & 92 [70-138] & $47-90 \mu \mathrm{mol} / \mathrm{L}$ \\
\hline Hs-Troponin T & $25(12-58.0)$ & $\leq 14 \mathrm{ng} / \mathrm{L}$ \\
\hline Total cholesterol & $3.8(2.76-4.72)$ & $<5 \mathrm{mmol} / \mathrm{L}$ \\
\hline Ferritin & 386 [149-830] & $15-150 \mu \mathrm{g} / \mathrm{L}$ \\
\hline РCT & $0.18(0.08-0.63)$ & $<0.1 \mu \mathrm{g} / \mathrm{L}$ \\
\hline HBA1C & $8.36 \pm 3.3$ & $<7 \%$ \\
\hline CRP & 84 [23-172] & $0-10 \mathrm{mg} / \mathrm{L}$ \\
\hline CD4 count & 210 [54-529] & $(500-2,010) \times 10^{5} / \mathrm{L}$ \\
\hline
\end{tabular}

Data are presented as mean \pm SD or median (IQR). CRP, C-reactive protein; HBA1C, glycated haemoglobin; INR, international normalised ratio; PCT, procalcitonin.

Table 3 Electrocardiographic features of the study patients

\begin{tabular}{lc}
\hline ECG abnormality & Values (total $n=200)$ \\
\hline Rhythm & $126(63 \%)$ \\
Sinus tachycardia & $10(5 \%)$ \\
Sinus bradycardia & \\
Dysrhythmias & $14(7 \%)$ \\
Atrial fibrillation & $4(2 \%)$ \\
Atrial flutter & $2(1 \%)$ \\
Supraventricular tachycardia & $1(0.5 \%)$ \\
Ventricular tachycardia & $6(3 \%)$ \\
Ventricular premature beats & \\
Chamber enlargement & $103(51.5 \%)$ \\
Left ventricular hypertrophy & $26(13 \%)$ \\
Right ventricular hypertrophy & $28(14 \%)$ \\
Left atrial enlargement & $14(7 \%)$ \\
Right atrial enlargement & \\
\hline
\end{tabular}

Table 3 (continued)
Table 3 (continued)

\begin{tabular}{lc}
\hline ECG abnormality & Values (total $n=200)$ \\
\hline Conduction system disease & \\
Right bundle branch block & $13(6.5 \%)$ \\
Left bundle branch block & $6(3.0 \%)$ \\
Left anterior and posterior fascicular & $7(3.5 \%)$ \\
block & \\
Complete heart block & $2(1 \%)$ \\
Sinus node dysfunction & $2(1 \%)$ \\
Intraventricular conduction delay & $9(4.5 \%)$ \\
Repolarisation abnormalities & \\
Early repolarisation abnormality & $26(13 \%)$ \\
Prolonged QT interval & $2(1 \%)$ \\
Poor R wave progression & $11(5.5 \%)$ \\
ST segment depression & $46(23 \%)$ \\
ST segment elevation & $7(3.5 \%)$ \\
T wave inversion & $29(14.5 \%)$ \\
\hline
\end{tabular}



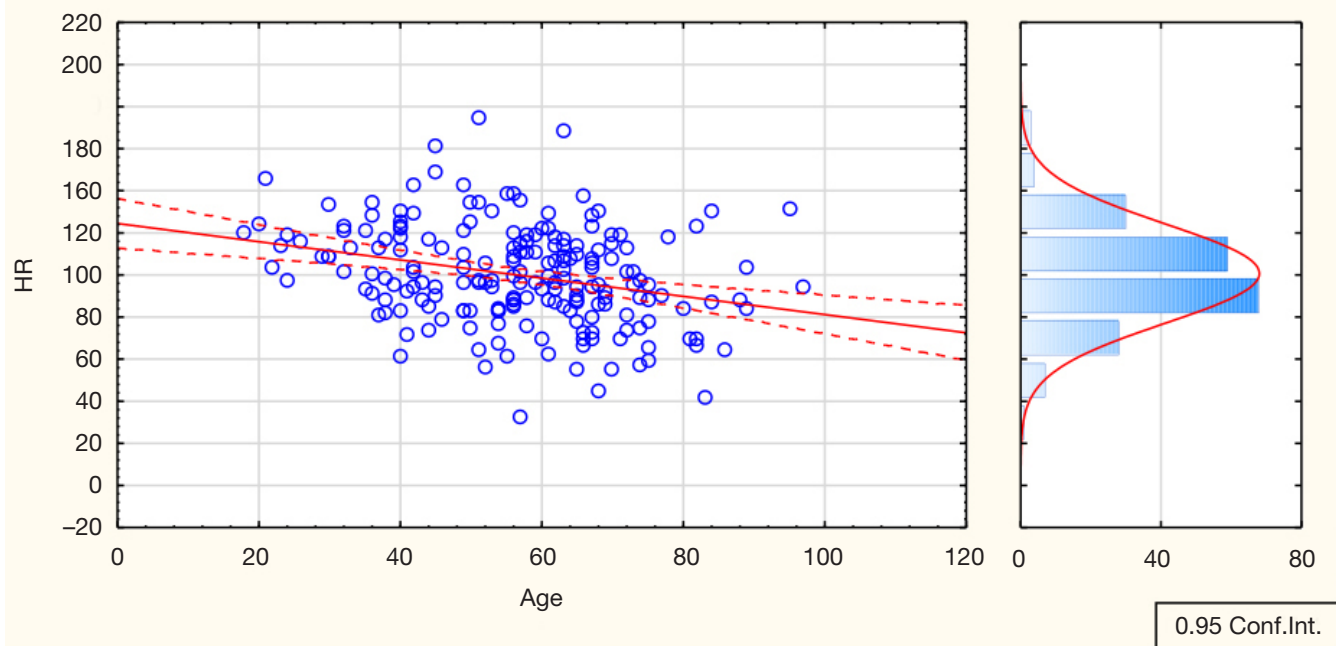

Figure 1 Correlation between age (years) and heart rate (HR) in patients with COVID-19 and cardiac disease $(\mathrm{r}=-0.28, \mathrm{P}=0.000)$.

Table 4 Echocardiographic characteristics of study patients

\begin{tabular}{lc}
\hline Echocardiography & Values (total n=200) \\
\hline Left ventricle & $21(10.5 \%)$ \\
Dilated left ventricle & $113(56.5 \%)$ \\
Concentric left ventricle hypertrophy & $21(10.5 \%)$ \\
Eccentric left ventricle hypertrophy & $100(50 \%)$ \\
Normal ejection fraction (>50\%) & $57(28.5 \%)$ \\
Mid-range ejection fraction (40-50\%) & $43(21.5 \%)$ \\
Low ejection fraction (<40\%) & $90(45 \%)$ \\
Left atrial enlargement & \\
Right ventricle & $118(59 \%)$ \\
Right ventricle enlargement & $91(45.5 \%)$ \\
Pulmonary hypertension & $72(36 \%)$ \\
Right atrial enlargement & $84(42 \%)$ \\
Biventricular enlargement & \\
Valvular heart disease & $109(54.5 \%)$ \\
Tricuspid regurgitation & $42(21 \%)$ \\
Mitral regurgitation/stenosis & $36(18 \%)$ \\
Aortic regurgitation/stenosis & \\
Congenital heart disease & \\
Atrial septal defect & $(1.5 \%)$ \\
Reprtial atrial ventricular canal defect & \\
Left sided infective endocarditis & \\
\hline
\end{tabular}

disease $(24.5 \%)$, right heart failure $(13 \%)$, abnormal electrocardiogram $(10 \%)$, arrythmias $(7.5 \%)$, acute coronary syndrome (7\%), suspicion of pulmonary embolism (4\%) and infective endocarditis (4\%). Echocardiography changed management in $53 \%$ of cases.

\section{The main echocardiographic findings}

The main echocardiographic findings are represented in Table 4. Twelve patients had normal echocardiograms and were excluded for the purpose of the current study. Three were excluded due to very poor imaging quality. The ejection fraction (EF) was preserved in $50 \%$ of cases. Very low EF was noted in $21.5 \%$ of cases. The most common findings were left ventricular hypertrophy or enlargement (77.5\%), followed by right ventricular enlargement (59\%). Hypertrophic cardiomyopathy was noted in two patients. Tricuspid valve regurgitation (TR) was noted in $54.5 \%$ of cases and four patients had organic TR. Mitral valve disease (MVD) was present in $42(21 \%)$ patients. Six patients had organic MVD. Aortic valve disease (AVD) was noted in 36 (18\%) patients, 14 had organic AVD. Features in keeping with pulmonary hypertension were present in $45.5 \%$ of patients. Global hypokinesia was noted in $26.5 \%$ of cases. Regional wall motion abnormality was present in $6(3 \%)$ of patients. Twenty- five patients $(12.5 \%)$ had pericardial effusions of which one presented in cardiac tamponade. One case of constrictive pericarditis was noted.

The most notable causes of heart failure were hypertensive heart disease with preserved ejection fraction 
(35.8\%), cardiomyopathies (20\%), cor pulmonale (15.7\%), infective endocarditis $(5.5 \%)$ and valvular heart disease (2.5\%). See Figures 2-4 for diagnosis post echocardiography in the study group.

\section{In-hospital outcome of patients with COVID-19 and cardiac disease}

In this case series $35(17.5 \%)$ patients died of which 21 $(60 \%)$ were males. The main cause of death was severe covid pneumonia in patients with underlying hypertensive heart disease $(\mathrm{n}=11)$, cor pulmonale due to severe covid pneumonia and PTED $(\mathrm{n}=6)$, heart failure due to underlying cardiomyopathy and covid pneumonia $(\mathrm{n}=5)$, conduction system disease and severe covid pneumonia $(n=4)$, infective endocarditis and covid ( $\mathrm{n}=3)$, acute coronary syndrome and covid pneumonia $(\mathrm{n}=3)$, partial $\mathrm{AV}$ canal defect and severe covid pneumonia $(\mathrm{n}=1)$, Hodgkin's lymphoma and covid pneumonia $(\mathrm{n}=1)$ and rheumatic mixed mitral valve disease and severe covid pneumonia $(\mathrm{n}=1)$. Four patients underwent successful cardiac surgery. The remainder of the patients were treated and discharged.

Patients who died had higher troponin levels and C-reactive protein (CRP) compared to those who survived (Table 5). There were no other laboratory differences noted between deceased and surviving patients.

In univariate analysis higher $\mathrm{SaO}_{2}$ was associated with lower risk of mortality (OR 1.02, 95\% CI: 1.00-1.05, $\mathrm{P}=0.046)$ (Table 6). Higher CRP was associated with increased risk of dying (OR 1.0, 95\% CI: 1.001-1.006, $\mathrm{P}=0.04)$. There were no statistically significant associations between mortality and the remaining patient characteristics such as obesity, troponins, hypertension, diabetes mellitus, ACEI/ARB, ejection fraction, pulmonary hypertension and sinus bradycardia $(\mathrm{P}>0.05)$.

In multivariable logistic regression (Table 6), there were no associations between patient characteristics and mortality except for sinus tachycardia and CRP. Sinus tachycardia emerged as the most important predictor of mortality in multivariate analysis. The presence of sinus tachycardia when adjusted for other factors increased the odds of dying 2.5 times. CRP was associated with a $1 \%$ increase in death for every unit increase in CRP (OR 1.0, 95\% CI: 1.0-1.02, $\mathrm{P}=0.02)$.

\section{Discussion}

This is the first South African study to have described the demographic, clinical, electrocardiographic and echocardiographic characteristics of an African population with COVID-19 and confirmed cardiac disease.

Our patients overall, were younger compared to western and Chinese populations afflicted with COVID-19 $(2,11)$. Most patients were obese females of African ancestry and had numerous co-morbidities. Fewer females were hospitalised in the study by Rossi et al., in contrast to this study. This may be explained by higher health seeking behaviour amongst females in our population. Further, mortality amongst males has been reported to be higher than females and we observed a similar trend in this study, though it failed to reach statistical significance. Geldsetzer et al. studied country-level data on COVID-19 deaths from the COVerAGE data base for countries for which age- and sex-disaggregated data were available (12). They proposed that higher mortality in males due to COVID-19 was likely due to a combination of biological, behavioural, and social pathways.

Hypertension was a common finding. This finding concurred with studies from China and Italy $(2,11)$. Hypertension was noted in in $50.4 \%$ of patients in a study by Shi $e t a l$. and $65.1 \%$ of hospitalized patients in a study by Giorgi Rossi et al. Hypertension has been shown to be associated with higher susceptibility to SARS-CoV-2 infection and adverse outcomes such as death (13). We did not find such an association between hypertension and mortality in this study (OR $1.33,95 \%$ CI: $0.58-0.34$, $\mathrm{P}=0.49$ ). However, in the group that died, hypertensive heart disease was noted in one third of patients. Further, recently use of renin-angiotensin-aldosterone (RAAS) blocking drugs was questioned in COVID-19 infection (13). It was postulated that blockade of RAAS pathway may worsen the outcomes in COVID-19 through upregulation of ACE 2 receptors. Use of ACEI/ARB was common in this study as majority of patients were hypertensive, but no difference was noted in their use between the groups that survived and died. Akin to the study by Giorgi Rossi et al. ACEI/ARB use did not predict mortality in this study (OR 0.97, 95\% CI: 0.46-2.02, $\mathrm{P}=0.93$ ) (11).

Obesity has been shown to be associated with adverse outcomes in patients with COVID-19 (14). However, in this study there was no difference in the prevalence of obesity and patient survival, and as such was not an important predictor of mortality. This finding needs to be interpreted with caution as presence or absence of obesity was assessed qualitatively by the attending physician.

Sinus tachycardia is a common response to systemic 

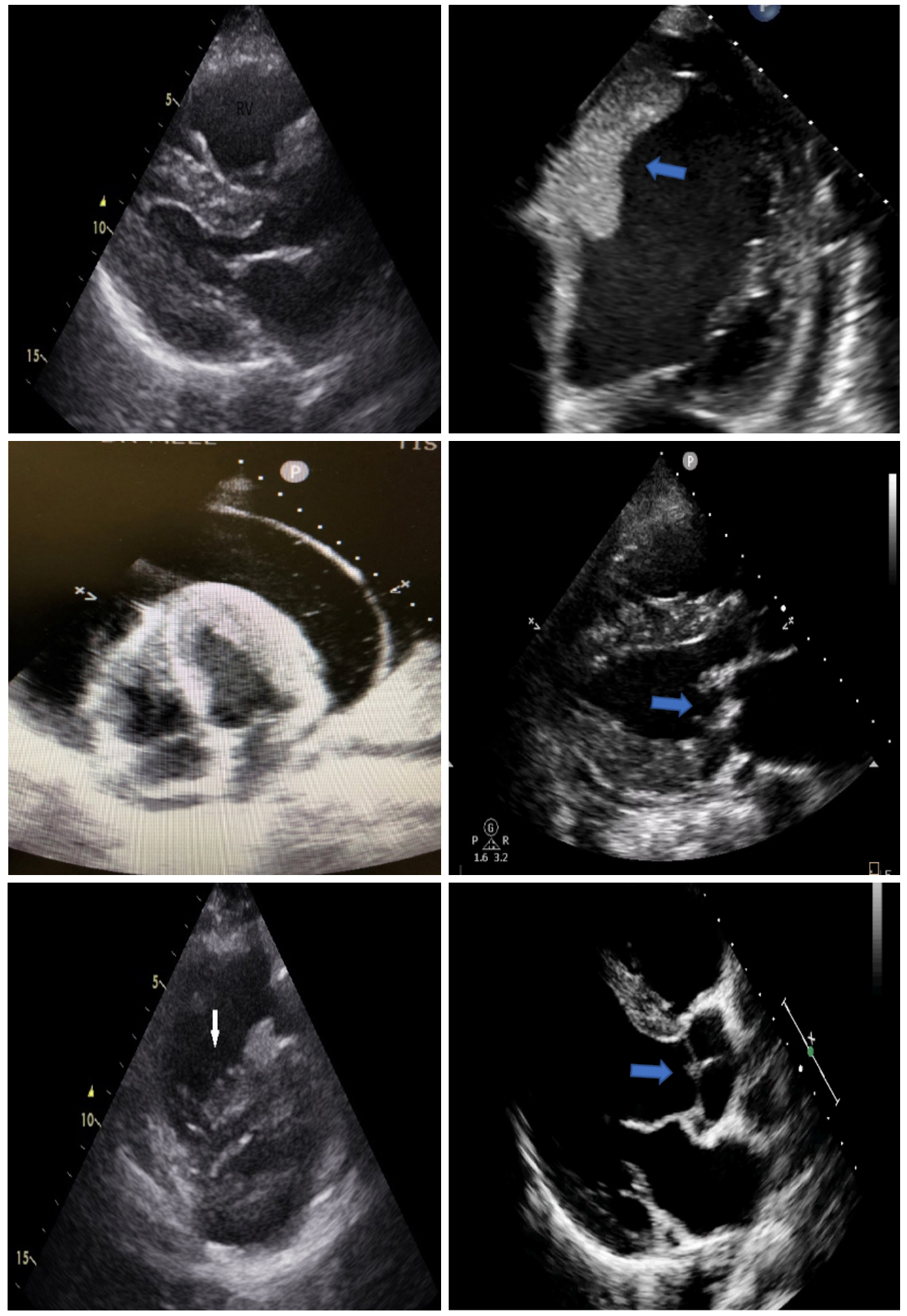

Figure 2 Echocardiographic images of patients with COVID-19 and cardiac disease. Top panel: patient with left ventricular hypertrophy and enlarged right ventricle (left) and dilated left ventricle (right). Middle panel: patient with pericardial effusion and tamponade (left) and rheumatic heart disease of the mitral valve (arrow) (right). Bottom panel: patient with severe pulmonary hypertension and reverse Berenheim effect (arrow) (left) and aortic valve infective endocarditis complicated by root abscess (arrow) (right). 

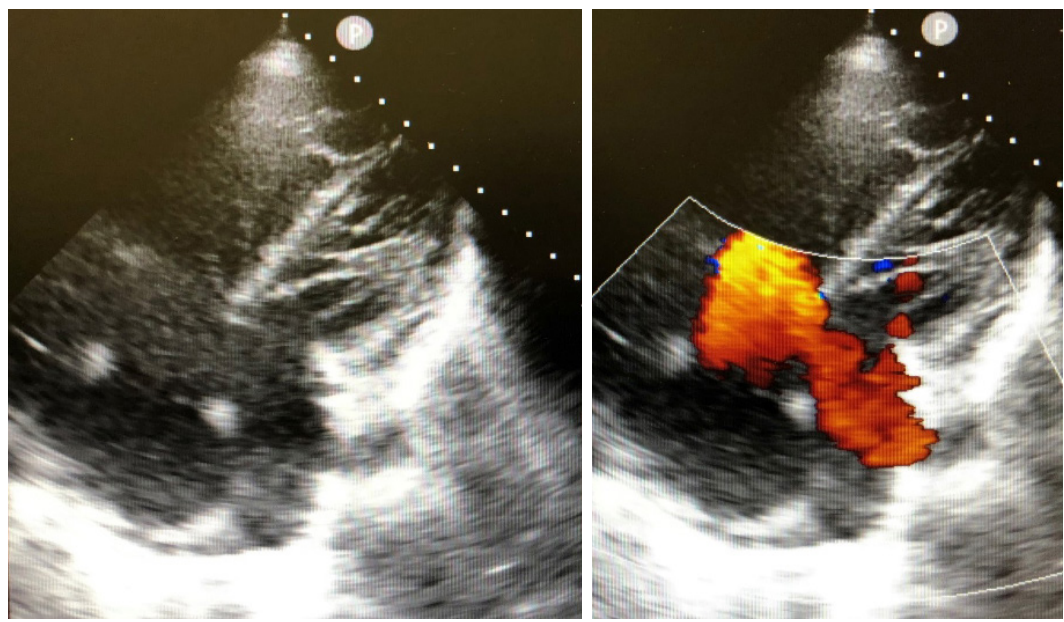

Figure 3 Transthoracic echocardiographic images of a severely ill patient with covid pneumonia and partial atrio-ventricular canal defect (arrow) complicated by significant right ventricle enlargement and pulmonary hypertension, thus exaggerating right to left shunting in a hypoxic patient.

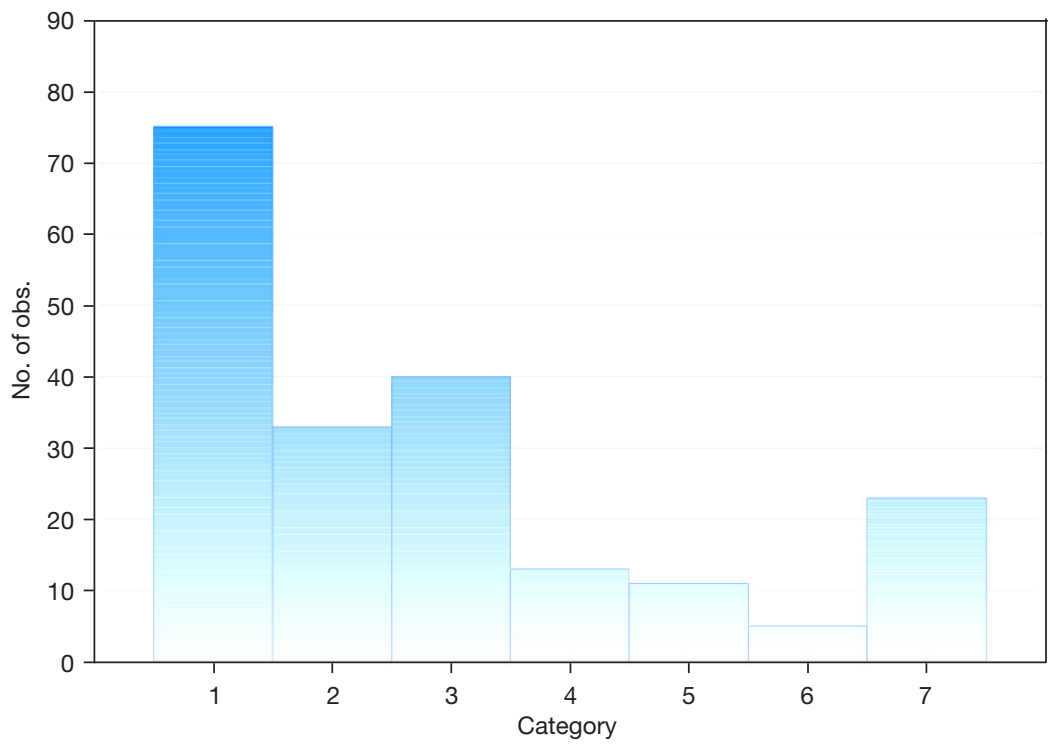

Figure 4 A frequency histogram with diagnostic category on the $\mathrm{x}$-axis and number of observations on the Y-axis. Diagnosis post echocardiography in patients with COVID-19 and suspected cardiac disease (1: hypertensive heart disease, 2: cor-pulmonale, 3: cardiomyopathy, 4: acute coronary syndrome, 5: infective endocarditis, 6: organic valvular heart disease, 7: suspected myocarditis, Tetralogy of Fallot, atrial septal defect, ascending aortic aneurysm, hypertrophic cardiomyopathy, constrictive pericarditis, cardiac tamponade, partial AV canal defect, electrical wall motion abnormalities).

infection or inflammation. In contrast to the study by Shi et al. (2), a high heart rate was an important predictor of mortality on multivariate analysis. Our findings concur with a study conducted in the US by Cho et al., whereby presence of sinus tachycardia was associated with lower survival in patients with COVID-19 (15). In a minority of cases, we noted sinus bradycardia. Sinus bradycardia in the context of COVID-19 infection is likely related to proinflammatory state, hypoxia, electrolyte imbalance, drugs and may be a precursor to a heightened cytokine storm $(16,17)$. In this study sinus bradycardia was encountered in individuals greater than age 50 years and is 
Table 5 Overall characteristics of cardiac patients by mortality

\begin{tabular}{|c|c|c|c|c|}
\hline Patient characteristics & All patients & Survivors & Non-survivors & $P$ value \\
\hline \multicolumn{5}{|l|}{ Demographic characteristics } \\
\hline Age, years & $56.47 \pm 15.63$ & $55.84 \pm 16.05$ & $59.43 \pm 13.29$ & 0.218 \\
\hline \multicolumn{5}{|l|}{ Gender, n (\%) } \\
\hline Female & $115(57.50)$ & 99 (86.09) & $16(13.91)$ & \\
\hline \multicolumn{5}{|l|}{ Clinical features } \\
\hline \multicolumn{5}{|l|}{ Co-morbidities } \\
\hline \multicolumn{5}{|l|}{ Body habitus } \\
\hline Overweight/metabolic syndrome & $47(23.50)$ & 39 (82.98) & $8(17.02)$ & \\
\hline Pre-existing cardiac disease & $26(13.00)$ & $23(88.46)$ & $3(11.54)$ & 0.581 \\
\hline Pre-existing lung disease & $36(18.00)$ & $30(83.33)$ & $6(16.67)$ & 0.884 \\
\hline HIV & $45(22.50)$ & 37 (82.22) & $8(17.78)$ & 0.956 \\
\hline Hypertension & $139(69.50)$ & $113(81.29)$ & $26(18.71)$ & 0.498 \\
\hline Diabetes mellitus & $63(31.50)$ & $53(84.13)$ & $10(15.87)$ & 0.681 \\
\hline Dyspnoea & $177(88.50)$ & $147(83.05)$ & 30 (16.95) & 0.570 \\
\hline \multicolumn{5}{|l|}{ Medications } \\
\hline Angiotensin converting enzyme inhibitors & $87(43.50)$ & $72(82.76)$ & $15(17.24)$ & 0.933 \\
\hline Diuretics & $127(63.50)$ & $103(81.10)$ & $24(18.90)$ & 0.493 \\
\hline Anti-diabetics & $50(25.00)$ & $38(76.00)$ & $12(24.00)$ & 0.162 \\
\hline \multicolumn{5}{|l|}{ Examination findings } \\
\hline Systolic blood pressure $(\mathrm{mmHg})$ & 129 [112-150] & 128 [112-149] & $129[112-160]$ & 0.555 \\
\hline Diastolic blood pressure $(\mathrm{mmHg})$ & $77.5(65-90.5)$ & 78 [65-91] & 77 [61-85] & 0.262 \\
\hline Heart rate beats/min & $100.07 \pm 23.45$ & $100.8 \pm 22.17$ & $96.63 \pm 28.89$ & 0.341 \\
\hline Respiratory rate (breaths/min) & 22 [20-25] & 22 [20-25] & 24 [20-25] & 0.111 \\
\hline Temperature $\left({ }^{\circ} \mathrm{C}\right)$ & $36.5(36.2-36.85)$ & $36.5(36.2-36.8)$ & $36.5(36.2-36.9)$ & 0.811 \\
\hline Saturation of oxygen (\%) & 87 [77-92] & 87 [78-92] & 85 [62-91] & 0.208 \\
\hline
\end{tabular}

Table 5 (continued) 
Table 5 (continued)

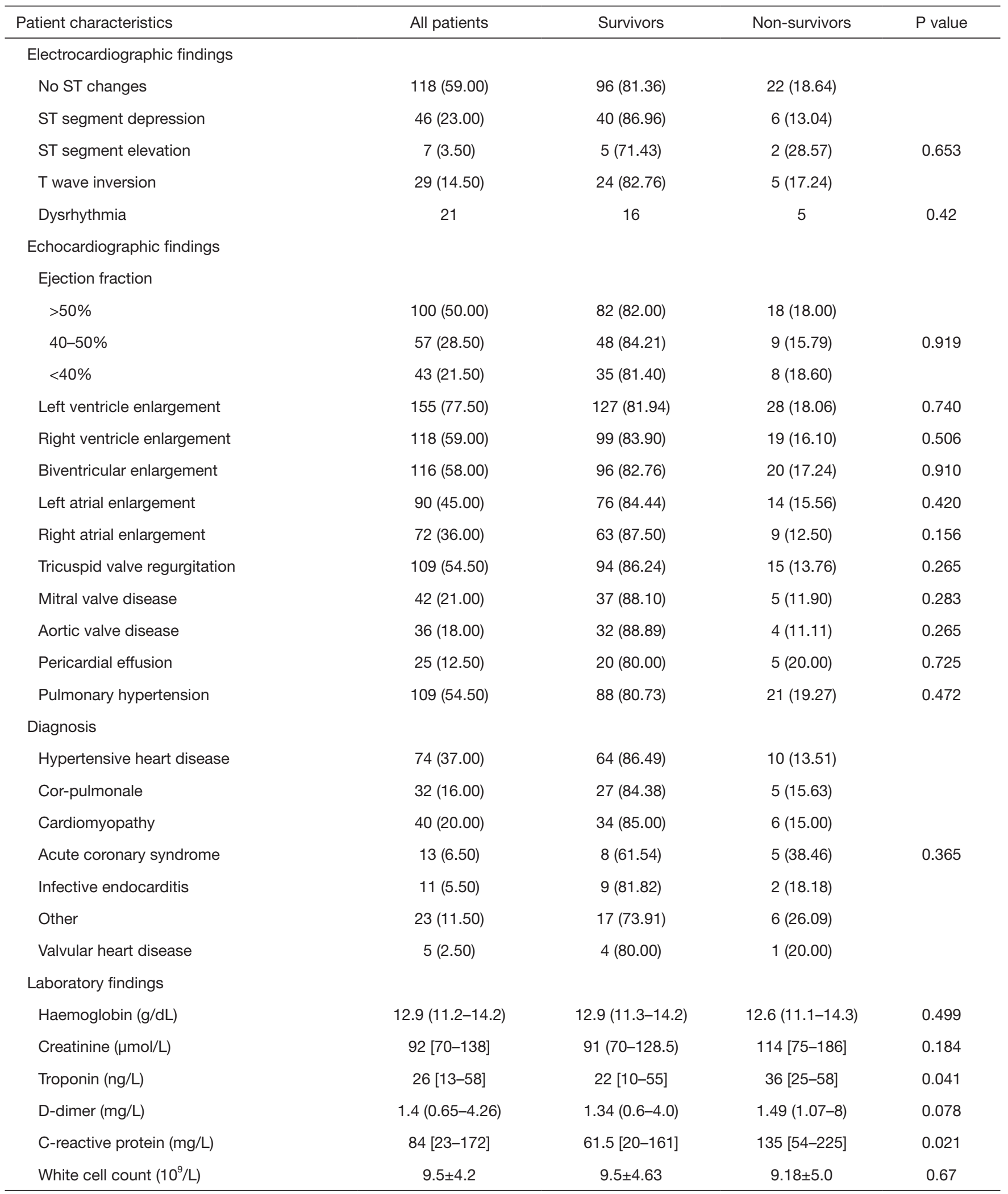

Data are presented as mean \pm SD or median (IQR) or $\mathrm{n}(\%)$. 
Table 6 Predictors of mortality in patients with cardiac disease and COVID-19

\begin{tabular}{|c|c|c|c|c|c|c|}
\hline Variable & \multicolumn{3}{|c|}{ Univariate logistic regression } & \multicolumn{3}{|c|}{ Multivariate logistic regression } \\
\hline Age (years) & 0.98 & $0.96-1.00$ & 0.218 & 1.00 & $0.98-1.03$ & 0.60 \\
\hline Gender (male) & 1.78 & $0.85-3.71$ & 0.123 & 0.50 & $0.23-1.11$ & 0.09 \\
\hline Systolic blood pressure (mmHg) & 0.99 & $0.98-1.01$ & 0.62 & 1.0 & $0.99-1.02$ & 0.22 \\
\hline Saturation of Oxygen (\%) & 1.02 & $1.00-1.05$ & 0.04 & 0.97 & $0.94-1.08$ & 0.08 \\
\hline C-reactive protein (mg/L) & 1.00 & $1.001-1.006$ & 0.04 & 1.01 & $1.00-1.02$ & 0.02 \\
\hline
\end{tabular}

likely due to a combination of disease of conduction system and superimposed inflammation. In the current study four patients that suffered sinus node disease and complete heart block with concurrent COVID-19 infection died.

Atrial fibrillation was the most common arrhythmia in this study. Most patients were older individuals with underlying comorbidities such as hypertension. Our findings are consistent with the studies by Antwi-Amoabeng et al. (18). AF is likely triggered by severe inflammatory response to SARS-Cov-2 infection and therefore, more a marker of severe underlying disease rather than a direct effect of SARS-CoV-2 virus. Atrial fibrillation with rapid ventricular response has been shown to be a predictor of mortality in COVID-19 (18). We did not find a significant difference in the prevalence of AF between the surviving and deceased groups.

Low oxygen saturation is an important contributor to hospital mortality (19). Hypoxia was an important contributor to mortality in univariate analysis in this study but failed to reach statistical significance in multivariate analysis. A study in Peru found an oxygen saturation less than $90 \%$ to be a strong predictor of in-hospital mortality, emphasising the need for timeous identification and treatment of hypoxaemia in patients with COVID-19 (19). Akin to this study all patients reported at least one co-morbidity.

Biomarkers of inflammation, coagulation and myocardial injury such as C-reactive protein (CRP), D-Dimer and hs-Troponin $\mathrm{T}$ were elevated in this study. CRP and hsTroponin were higher in the non-survivors compared to survivors, implying that a heightened inflammatory state likely existed in this group of patients. This finding was corroborated in a meta-analysis by Alzahrani et al., where levels of biomarkers such as troponin $\mathrm{T}$ and CRP were higher in non-survivors than survivors (20). Patients with higher levels of biomarkers were at increased risk of mortality. D-dimer levels were higher in the deceased group but failed to reach statistical significance. In our study only CRP was associated with increased risk of death in univariate and multivariate analysis.

In this study we used handheld echocardiography as bedside tool for qualitative assessment of cardiac structure and function. Similar to prior experience with this mode of imaging of COVID patients the author found handheld echocardiography to be highly portable, easy to disinfect and an ideal imaging tool for rapid scanning of these highly infectious patients (9). Left and right ventricular involvement was noted in over half of patients. This was higher than the global evaluation of echocardiography in patients with COVID-19 by Dweck et al. (10). Similar to the aforementioned study heart failure was a frequent indication for echocardiography in this study. Prevalence of severe left ventricular dysfunction was higher in this study at $21 \%$ compared to $9 \%$ in Dweck et al. study. As noted in our study, hypertension was a common co-morbidity similar to the study by Dweck et al. However, they did not specifically report on left ventricular hypertrophy, which was a common finding in the current study. Myocarditis was suspected in two cases in this study but true assessment was confounded by co-existence of underlying HIV and peripartum cardiomyopathy in these patients and lack of diagnostic cardiac magnetic resonance imaging. We did not encounter any cases of classic takotsubo cardiomyopathy in this study. However, this diagnosis may have been underestimated in patients with globally hypokinetic dilated ventricles where it was likely attributed to dilated cardiomyopathy or possible myocarditis.

In contrast to the global evaluation of echocardiography (10) in patients with COVID-19, echocardiography changed management in half of the current study patients. This may be explained by design of the current study whereby 
only patients with cardiac disease were studied and smaller sample size that included the sickest patients with multiple co-morbidities. Further, in contrast to the study by Dweck et al. all these patients were seen, scanned and managed by a single experienced Cardiologist. Echocardiography accompanied with good multi-disciplinary clinical assessment allowed focused management of the patient's diseases. These included conditions such as infective endocarditis, pericardial effusion with tamponade, pulmonary embolism, acute coronary syndromes and titration of heart failure therapy. Dweck et al. reported a similar change in patients management in their study.

Finally, the in-hospital mortality rate in this study was $17.5 \%$ which falls within the overall internationally reported range of $13.2 \%$ to $28.3 \%$ of patients with COVID-19 infection and just below internationally reported rates of $24.2 \%$ of patients with cardiac disease and COVID-19 subgroup (2). A meta- analysis by Cordero et al. of patients with cardiovascular disease and mortality reported that those with cardiovascular disease and COVID-19 had 4-fold higher risk of death (21). Further, it was highlighted that mortality rates reported in hospital registries and national reports were even higher at $48.7 \%$ and $23.1 \%$ respectively. The mortality amongst patients with cardiovascular disease (CVD) in this study is likely an underestimate as we did not systematically include all patients with possible CVD admitted to hospital. However, this study group comprised patients that required cardiac consultations and hence is representative of sickest patients with cardiac disease and COVID-19 infection.

\section{Limitations}

This was a retrospective single centre study and as such suffers from the usual limitations associated with such a study design. The findings of this study may not be extrapolated to other population groups due to biologic differences in age, gender, race and genetic factors amongst patients with cardiac disease and COVID-19. Only qualitative echocardiography data was recorded on most patients due to the infectious nature of the disease. Echocardiography was performed by a single operator and this may have introduced unintended bias. The study is limited to patients with confirmed cardiac disease on echocardiography and therefore suffers from selection bias. Additional imaging modality such as cardiac MRI, invasive coronary angiography and coronary computed tomography were not performed.

\section{Conclusions}

The South African patient with COVID-19 and cardiac disease are predominantly obese females with underlying hypertension. Handheld echocardiography is a useful tool in the setting of COVID-19 disease and cardiac disease. Mortality amongst this select group of patients was high and they were predominantly males. Hypoxia and raised CRP were important predictors of mortality in this study, however, sinus tachycardia emerged as the most important independent predictor of in-hospital mortality.

\section{Acknowledgments}

Funding: The study was supported by Post-Doctoral Carnegie Fund.

\section{Footnote}

Reporting Checklist: The authors have completed the STROBE reporting checklist. Available at https://dx.doi. org/10.21037/cdt-21-459

Data Sharing Statement: Available at https://dx.doi. org/10.21037/cdt-21-459

Peer Review File: Available at https://dx.doi.org/10.21037/ cdt-21-459

Conflicts of Interest: All Authors have completed the ICMJE uniform disclosure form (available at https://dx.doi. org/10.21037/cdt-21-459). The authors have no conflicts of interest to declare.

Ethical Statement: The authors are accountable for all aspects of the work in ensuring that the accuracy or integrity of any part of the work are appropriately investigated and resolved. The study was conducted in accordance with the Declaration of Helsinki (as revised in 2013). The study was approved by the ethics committee of University of the Witwatersrand (M200678). Because of the retrospective nature of the study, the requirement for informed consent was waived.

Open Access Statement: This is an Open Access article distributed in accordance with the Creative Commons Attribution-NonCommercial-NoDerivs 4.0 International License (CC BY-NC-ND 4.0), which permits the non- 
commercial replication and distribution of the article with the strict proviso that no changes or edits are made and the original work is properly cited (including links to both the formal publication through the relevant DOI and the license). See: https://creativecommons.org/licenses/by-nc-nd/4.0/.

\section{References}

1. Zhou F, Yu T, Du R, et al. Clinical course and risk factors for mortality of adult inpatients with COVID-19 in Wuhan, China: a retrospective cohort study. Lancet 2020;395:1054-62.

2. Shi S, Qin M, Shen B, et al. Association of Cardiac Injury With Mortality in Hospitalized Patients With COVID-19 in Wuhan, China. JAMA Cardiol 2020;5:802-10.

3. Wu Z, McGoogan JM. Characteristics of and Important Lessons From the Coronavirus Disease 2019 (COVID-19) Outbreak in China: Summary of a Report of 72314 Cases From the Chinese Center for Disease Control and Prevention. JAMA 2020;323:1239-42.

4. Wang D, Hu B, Hu C, et al. Clinical Characteristics of 138 Hospitalized Patients With 2019 Novel CoronavirusInfected Pneumonia in Wuhan, China. JAMA 2020;323:1061-9.

5. Meel R, Gonçalves R. Overview of cardiovascular manifestations of COVID-19 and echocardiographic features. SA Heart 2020;17:200-3.

6. Meel R, Ramsamy TD, Narsing R, et al. Focal myocarditis in a young male with SARS-CoV-2 infection. Oxf Med Case Reports 2021;2021:omaa142.

7. Ackermann M, Verleden SE, Kuehnel M, et al. Pulmonary Vascular Endothelialitis, Thrombosis, and Angiogenesis in Covid-19. N Engl J Med 2020;383:120-8.

8. Meel R, Cupido B, Pecoraro A, et al. COVID-19 and cardiovascular imaging: A guide for the practising clinician. SA Heart 2020;17:306-12.

9. McMahon SR, De Francis G, Schwartz S, et al. TabletBased Limited Echocardiography to Reduce Sonographer Scan and Decontamination Time during the COVID-19 Pandemic. J Am Soc Echocardiogr 2020;33:895-9.

10. Dweck MR, Bularga A, Hahn RT, et al. Global evaluation of echocardiography in patients with COVID-19. Eur Heart J Cardiovasc Imaging 2020;21:949-58.

11. Giorgi Rossi P, Marino M, Formisano D, et al. Characteristics and outcomes of a cohort of COVID-19 patients in the Province of Reggio Emilia, Italy. PLoS One 2020;15:e238281.
12. Geldsetzer P, Mukama T, Jawad NK, et al. Sex differences in the mortality rate for coronavirus disease 2019 compared to other causes of death. Available online: https://www.medrxiv.org/content/10.1101/2021.02.23.212 52314v1.full.pdf

13. Savoia C, Volpe M, Kreutz R. Hypertension, a Moving Target in COVID-19: Current Views and Perspectives. Circ Res 2021;128:1062-79.

14. Kompaniyets L, Goodman AB, Belay B, et al. Body Mass Index and Risk for COVID-19-Related Hospitalization, Intensive Care Unit Admission, Invasive Mechanical Ventilation, and Death - United States, MarchDecember 2020. MMWR Morb Mortal Wkly Rep 2021;70:355-61.

15. Cho JH, Namazi A, Shelton R, et al. Cardiac arrhythmias in hospitalized patients with COVID-19: A prospective observational study in the western United States. PLoS One 2020;15:e0244533.

16. Amaratunga EA, Corwin DS, Moran L, et al. Bradycardia in Patients With COVID-19: A Calm Before the Storm? Cureus 2020;12:e8599.

17. Hu L, Gong L, Jiang Z, et al. Clinical analysis of sinus bradycardia in patients with severe COVID-19 pneumonia. Crit Care 2020;24:257.

18. Antwi-Amoabeng D, Beutler BD, Singh S, et al. Association between electrocardiographic features and mortality in COVID-19 patients. Ann Noninvasive Electrocardiol 2021;26:e12833.

19. Mejía F, Medina C, Cornejo E, et al. Oxygen saturation as a predictor of mortality in hospitalized adult patients with COVID-19 in a public hospital in Lima, Peru. PLoS One 2020;15:e244171.

20. Alzahrani SH, Al-Rabia MW. Cardiac Injury Biomarkers and the Risk of Death in Patients with COVID-19: A Systematic Review and Meta-Analysis. Cardiol Res Pract 2021;2021:9363569.

21. Cordero A, García-Gallego CS, Bertomeu-González V, et al. Mortality associated with cardiovascular disease in patients with COVID-19. REC: CardioClinics 2021;56:30-8.

Cite this article as: Meel R, Van Blydenstein SA. Demographic, clinical, electrocardiographic and echocardiographic characteristics of patients hospitalized with COVID-19 and cardiac disease at a tertiary hospital, South Africa. Cardiovasc Diagn Ther 2021;11(6):1228-1240. doi: 10.21037/cdt-21-459 